\title{
Development of ID-Blade Slicer Monitoring System for Cutting 12-inch Silicon Ingot
}

\author{
Zhongwei Jiang $^{* 1}$, Shusuke Fujiwara ${ }^{* 2}$, Seiji Chonan ${ }^{* 2}$ and Kazuo Kawashima ${ }^{* 3}$

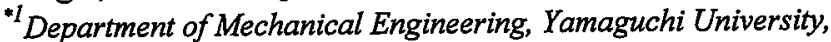 \\ 2-16-1 Tokiwadai, Ube, 755-8611 Yamaguchi, Japan \\ ${ }^{*}$ Tohoku University, ${ }^{* 3}$ Asahi Diamond Industrial Co. Ld.
}

\begin{abstract}
This work is concerned with an experimental study on online measurement of the states of ID saw blade cutting a 12-inch crystal ingot. An online monitoring system consisting of a 3-axis piezoelectric sensor, a three-band digital filter, a digital oscilloscope and computer was developed. Since the acoustical and chatter vibration signals during cutting ingot were complex, they are decomposed in three frequency regions to evaluate the blade extension circumstance, the sharpness of blade edge and the interference between blade and ingot. The proposed ID-Blade slicer monitoring system is validated for evaluation of the blade slicing 12 -inch silicon ingots.
\end{abstract}

\section{Introduction}

The market of semiconductor devices is growing so fast completely beyond the common prediction. In order to increase the productivity of semiconductor devices, the size of the crystal ingot goes bigger and bigger from 6 inches to 8 inches, recently achieved to 12 inches in diameter. In the manufacturing process of semiconductor devices, the ingot slicing is the first process and the surface quality of sliced wafer is the most important factor because it affects following processes severely. ID (inner-diameter) saw blade is commonly used for cutting the ingots and there are a lot of researches on the dynamic characteristics of ID blade ${ }^{(1)-(3)}$ The blade is electrodeposited by diamond abrasive grains at its inner edge about $3 \mathrm{~mm}$ in width, and tensioned initially at the outer periphery to increase its stiffness and to make the blade edge as circular as perfect. The size for 6-inch ingot blade is $0.665 \mathrm{~m}$ at outer diameter, $0.24 \mathrm{~m}$ at inner diameter and $0.13 \mu \mathrm{m}$ in thickness. As for 12-inch ingot the blade would be as twice large in diameter as 6-inch ingot blade but the thickness should not be changed too much. Some semiconductor makers even have believed that ID blade is not suitable for cutting 12-inch ingot until the first ID-Blade slicer for 12-inch ingot was developed by Toyo Advanced Technologies Co., Ltd and Asahi Diamond Industrial Co., Ltd. In this machine, the size of the blade is $1.18 \mathrm{~m}$ at outer diameter, $0.41 \mathrm{~m}$ at inner diameter and $0.18 \mu \mathrm{m}$ in thickness. An efficiency test validated that $\mathrm{D}$ Blade slicer is still a precise and efficient tool for slicing 12-inch ingot. However, since it comes twice large in diameter but almost same in thickness, the blade becomes more delicate and an advanced control system for accurate cutting is required if one wants to keep the wafer quality as same as in 6-inch wafer.

For building an accurate control system, the first problem that should be solved is how to measure and evaluate the cutting state of the blade online. In the present 12-inch wafer slicer, the blade edge deflection is monitored for controlling blade rotation and feeding speed of ingot. But it showed that the deflection is not enough for 12-inch ingot blade. In this study, an online monitoring system of the acoustical and chatter vibration signals was developed and the evaluation technique on the cutting state of blade was investigated.

\section{Online Measuring and Monitoring System}

Figure 1 shows the outline of the measuring system consisting of a 3-axis piezoelectric sensor, a threeband digital filter, a digital oscilloscope and computer. The slicer T-SM-300 used in experiment equipped with $\mathrm{U}-\mathrm{ABC}$ (ultra auto bow control) so that the warp of wafer can be easily controlled by moving the ingot in its axis direction(z-direction). The blade rotates normally around $1040 \mathrm{rpm}$ and the ingot is fed in $\mathrm{x}$-direction at $30 \mathrm{~mm} / \mathrm{min}$. In order to assess the cutting performances of the blade, the piezosensor is fixed onto the mount of the ingot. The signals, generated during slicing and passed through the ingot, are picked up by the sensor. Further, the signals involve the scraping sound as the diamond grain layer against the ingot, the chatter vibration from the blade edge and the vibration from blade rotation or slicer itself. Since the signal levers are different in each cases, the digital filter is introduced for extracting high $\mathrm{S} / \mathrm{N}$ ratio signals in three frequency regions, i.e., low pass to $2 \mathrm{kHz}$, high pass from $300 \mathrm{~Hz}$ and $1500 \mathrm{~Hz}$. Furthermore, the signals are sampled and recorded in the digital oscilloscope and then forwarded into the computer for analysis and display.

\footnotetext{
* Corresponding author: E-mail: jiang@po.cc.yamaguchi-u.ac.jp Fax: +81.836.85.9137
} 


\section{Evaluation of Blade State and Cutting Performance}

Figure 2 shows the detail of the cutting performance monitoring system and the blade evaluation concept. The signals both in $x$ - and $y$-directions are collected simultaneously and classified in three cases. Case 1 is for low range frequency signal obtained by low pass filter with sampling frequency $4 \mathrm{kHz}$. It is found that their main ingredient corresponds to the blade rotation and the cutting force. Case 2 is for middle range frequency signal in which the frequency components lower than $300 \mathrm{~Hz}$ were filtered out so that the desired signal could be amplified and recorded in the oscilloscope with a high resolution. The data in this case involves the information of the blade vibration and the circumstance of the diamond edge layer. In Case 3, the $1500 \mathrm{~Hz}$ high pass filter is introduced in order to obtain the acoustical signals as the blade scraping the ingot. These three cases are measured in successive turns controlled by the software.

The signals obtained in Case 1 is used for monitoring the cutting performance of the blade. The average value of $x$-direction signals is calculated and it indicates a good estimation of blade sharpness. Further, the signal in $y$-direction is disposed by FFT analysis and the peak values of its power spectrum are calculated. It is validated that the pattern of the peak values implies the effects of the elliptical shape at the blade inner edge, the distribution of in-plane stresses due to the initial extension, the centrifugal force and the cutting loads. The signal obtained in Case 3 contains mainly high frequency components around $4 \mathrm{kHz}$ to $8 \mathrm{kHz}$, which are also easily heard by human's ears and usually used by the operator to assess the present state of blade. In the experimental study it is discovered that the acoustical sound lever in this region is an important factor for monitoring the interference or scraping between the blade and ingot which could stimulate the vibration in the blade and break the wafer. The scraping phenomenon happened frequently to the case when the wafer is required to cut in warp. The scraping between the blade and ingot is not so serious problem for 6-inch ingot slicer. However, it is very severe problem for 12-inch ingot slicer. In case it could lead the wafer broken, damage the blade and even stop the manufacturing line. As for Case 2 , the signals are more complicate and involve a lot of information, such as the chatter vibration, the effect of diamond grain layer and so on. An advanced investigation on this issue will be discussed in our next report.

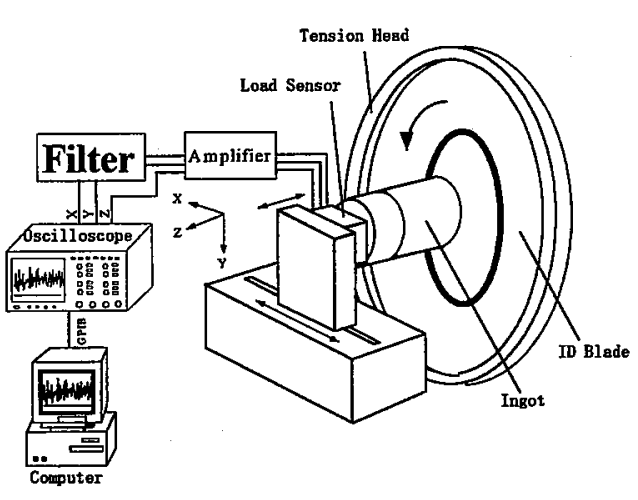

Fig. 1. Experimental setup for 3-directional acoustical and vibration signal measurement.

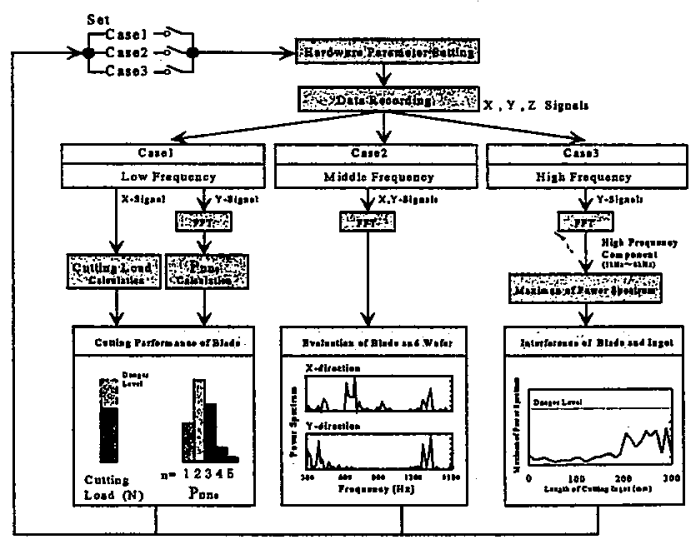

Fig. 2. Concept of online monitoring and evaluation system for cutting 12-inch ingot.

\section{Conclusions}

The online monitoring system was developed for evaluation of the blade state and the performance of cutting silicon ingots. The sound of scraping and vibration signals were measured by a 3-axis piezosensor and processed by computer. Several expressions were derived for assessment of the sharpness of blade, the elliptical shape at inner edge of blade, and the occurrence of the interference between blade and ingot. It is also indicated that the acoustical and vibration signals could be used as feedback signals so that a new accurate control system could be realized for the 12-inch ingot ID-Blade slicer.

\section{References}

[1] S.E. Forman, W.J. Rhines "Vibration Characteristics of Crystal Slicing ID Saw Blade," J. Electrochemical Society, No.119, 686-690, 1972

[2] K. Ishikawa, H. Suwabw, M. Uneda and N.Kurata, "Dynamical Study on ID-Blade Slcing Utilizing Elliptical Vibration: Mechanism of Elliptical Vibration Slicing and Behavior of Slicing Force," J. Japan Society for Precision Engineering, Vol.65 No.11, 1605-1610, 1999.

[3] Z.W.Jiang, S.Chonan, K.Kawashima, K.Muto and W.Ichhara, "Condition Monitoring of Sillicon-Wafer Slicer Cutting Crystal Ingots," Structural Health Monitoring, Technomic Publishing, 624-635, 1997. 